\title{
Smart City, tendencias y evolución: Un análisis bibliométrico.
}

\section{Smart City, trends, and evolution: A bibliometric analysis.}

\author{
Gabriel M. Martinez-Toro ${ }^{1}$, Gerly Carolina Ariza-Zabala ${ }^{2}$, María Monica Vargas-Mantilla ${ }^{3}$, Efrén Romero-Riaño ${ }^{4}$, \\ Dewar Rico-Bautista ${ }^{5}$ \\ ${ }^{1,2,3}$ Universidad Autónoma de Bucaramanga, Bucaramanga - Colombia, \\ ${ }^{4}$ Universidad Industrial de Santander, Bucaramanga - Colombia, \\ ${ }^{5}$ Universidad Francisco de Paula Santander Ocaña, Ocaña - Colombia

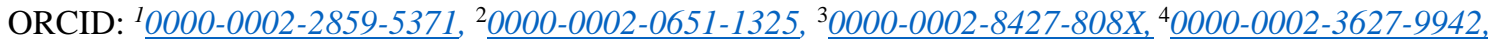 \\ ${ }^{5} 0000-0002-1808-3874$
}

Recibido: 17 de julio de 2020.

Aprobado: 11 de noviembre de 2020.

\begin{abstract}
Resumen - Una ciudad inteligente, es una ciudad que sitúa a las personas como centro de desarrollo e incorpora TICS en la gestión urbana como respuesta a problemas como la contaminación, inseguridad y congestión. Los países desarrollados se enfocan en mejorar la calidad de vida de sus habitantes y trabajan en hacer de las ciudades, espacios inteligentes, incluyentes y sostenibles. En el ámbito académico, el término "smart city" ha tenido un interés en aumento, como se demuestra en una búsqueda en la base de datos WoS de Clarivate, en la cual se registran 9 records para el 2011 incrementándose a 950 records en 2019. De los resultados de la búsqueda se obtuvo una muestra de 2833 documentos en el periodo entre 2001 - 2019. Al ser un área de conocimiento en desarrollo es relevante conocer las tendencias que enmarcan su evolución. El análisis propuesto se basa en los postulados de la bibliometría y la cienciometría, que por medio del examen de los patrones de coocurrencia de términos y de la evaluación de la fortaleza de vínculos entre componentes de una red, se infiere la existencia de grupos o clusters de conocimiento, se reconocen temáticas emergentes, temáticas en declive, publicaciones relevantes, entre otras. Para el procesamiento de datos se implementó la herramienta VOSviewer, el cual es un software gratuito para la visualización de redes de conocimiento. En el estudio se puede observar que el área de conocimiento se focaliza en 3 temáticas: 1) Desarrollo de ciudades a partir de la innovación y sostenibilidad, 2) diseño y administración de ciudades a través del internet e internet de las cosas y 3) marcos de trabajo basados en bigdata encaminados hacia la gobernanza. Estos resultados orientan a los investigadores interesados en ampliar los horizontes de esta temática y en buscar nuevos paradigmas relacionados con Smart city.
\end{abstract}

Palabras Clave: Análisis bibliométrico; Análisis de redes sociales; Análisis de coocurrencia; Ciudad inteligente.

Abstract - A smart city places people at the centre of development and incorporates ICTs into urban management as a response to problems such as pollution, insecurity, and congestion. Developed countries focus on improving the quality of life of their inhabitants and work on making cities intelligent, inclusive, and sustainable spaces. In academia, the term "smart city" has been gaining interest, as shown by a review of Clarivate's WoS database, which shows 9 records for 2011 rising to 950 records in 2019. The search results showed a sample of 2833 documents in the period from $2001-2019$. As an area of knowledge in development, it is relevant to know the trends that frame its evolution. The proposed analysis is based on the postulates of bibliometrics and scientometrics, which through the co-occurrence of data and the strength of links between components of a network, infers related bodies of knowledge in an objective manner, recognizing hot topics, declining themes, relevant publications, among others. The VOSviewer software was used as an information processing tool, which is a open source software that provides the construction and visualization of knowledge networks, which facilitates their exploration. In this study one can see that the knowledge area is focused on 3 themes: 1) Development of cities based on innovation and sustainability, 2) design and administration of cities by means of Internet and the Internet of Things as well as 3) frameworks based on bigdata aimed at governance. These results guide researchers interested in broadening the horizons of this theme and in seeking new paradigms related to the Smart city.

Keywords: Bibliometric analysis; Social network analysis; Co-word analysis; Smart City. 


\section{INTRODUCCIÓN}

A medida que la población y densidad de las ciudades aumenta, los retos y problemas del mundo son mayores; una ciudad inteligente Smart City (SC)- sitúa a las personas en el centro de desarrollo e incorpora las tecnologías de información y comunicaciones (TICs) en la gestión urbana como respuesta a los problemas que la afectan. Los países desarrollados se enfocan en mejorar la calidad de vida de sus habitantes y trabajan en hacer de estas, espacios inteligentes, incluyentes y sostenibles. Las grandes urbes tienen problemas y consumen muchos recursos que han venido afectando la sostenibilidad del planeta.

Desde [1], se cita a la Comunidad Europea, en cuyo informe Smart cities and communities Ciudades Inteligentes, define a estas ciudades como:

Sistemas de personas que interactúan y que utilizan los flujos de energía, materiales y financiación para catalizar el desarrollo económico sostenible, flexible y una alta calidad de vida; estos flujos e interacciones se vuelven inteligentes a través de hacer uso estratégico de la infraestructura y los servicios de información y comunicación en un proceso de planificación urbana y gestión transpa-rente que responde a las necesidades sociales y económicas de la sociedad.

El desarrollo de ciudades inteligentes presente y hacia el futuro ha tomado interés por gobiernos, académicos, tecnólogos y empresarios. De acuerdo con [2], se han construido cerca de 1000 SC en el mundo, en países de Europa, Norte América y Asia. Por lo tanto, los estudios e investigaciones relacionadas con el desarrollo y gestión de ciudades inteligentes también han aumentado.

El concepto de SC cobra importancia este momento que el mundo atraviesa la pandemia del coronavirus. El tamaño de las ciudades, las migraciones, la globalización y la tecnología de transporte aceleraron el contagio entre países, ciudades "enfermas" llevaron el aislamiento obligatorio para detener problemas. Esta situación ha evidenciado la dependencia de las TICs, siendo claves, no solo para mantener los lazos humanos, sino para que en las ciudades se pueda seguir trabajando, las actividades académicas continúen, operaciones financieras funcionen, entre otras, es decir para no parar la economía, ni a las empresas y así no atrasar el desarrollo de las ciudades y países. El transporte de mercancías, alimentos, medicamentos a nivel local, regional, nacional e internacionalmente también debe continuar y acelerarse. Los elementos de las ciudades inteligentes no se limitan a la infraestructura física, de transporte y a las TIC; involucran e-Gobierno y Gobierno Abierto (Open Data), computación en la nube, Big data y el internet de las cosas [3], pues no se trata de ciudades digitales, sino de ciudades que atienden las necesidades de personas y comunidades inteligentemente.

En ese orden de ideas, se justifica revisar la evolución y tendencias de las SC, a través de los diferentes estudios e investigaciones realizados en el tiempo. Se pretende que esta revisión sirva a otros investigadores en la planificación, diseño y tal vez, rediseño de SC.

La ubicación documental y el estado del arte del tema de SC es cada vez mayor, complicando la consulta de fuentes, autores, temáticas. Para hacer una revisión de forma objetiva y organizada de artículos, publicaciones y ponencias, se utiliza la cienciografía y luego se realiza un análisis bibliográfico [4] con el apoyo del software VOSviewer que permite la creación de mapas de datos de literatura científica.

A continuación, se presenta la metodología aplicada para la búsqueda, revisión y el análisis de la información extraída de la base de datos Web of Science. Por medio del uso del software libre VOS Viewer se construyen los mapas relacionados con el campo de conocimiento de las ciudades inteligentes (SC), en información ubicada a partir del año 2001 al 2019, (2833 datos) desde el punto de vista de términos claves y se realiza el análisis de la coocurrencia de a partir de metadatos y clústeres identificados.

Finalmente, se presentan los resultados en cuanto a lo más tratado, los aspectos que han analizado y la identificación de elementos que no se ha abordado o apenas se han iniciado, en lo que apunta al concepto integral de SC. Se identifican las tendencias a investigar y las oportunidades de estudio para los investigadores, empresas, gobiernos, ONGs y paises en alianza; todo en bien de la calidad de vida, la convivencia en comunidades urbanas y la sostenibilidad futura de las ciudades.

\section{MARCO TEÓRICO}

Las ciudades tienen un rol clave en el desarrollo de la humanidad, siendo las necesidades planteadas por la vida urbana el eje central de este desarrollo. Soportar la toma de decisiones inteligentes, en un ambiente en el que el tiempo real propone fecha de vencimiento a la información, plantea retos que se encaminen a facilitar el uso de los servicios y mejorar la calidad de la vida urbana [5]. Hay que tener presente que más de la mitad de la población habita en las ciudades, ciudades multiculturales y vibrantes, que deben ser gobernadas de manera incluyente e innovativa siguiendo enfoques de tecnología, educación y colaboración que las haga mas inteligentes y que facilite el desarrollo de valores públicos [6].

El modelo urbano de SC es uno de los más difundidos y de amplia aceptación, en parte por su naturaleza sistémica y multidimensional, construida con base en la participación de los ciudadanos. El modelo de SC propuesto por [7], compone de seis aspectos urbanos principales: Gobernanza, Gente, Economía, Medio Ambiente, Vivienda y Movilidad. Estas ciudades inteligentes se diferencian por ofrecer servicios a sus habitantes con el fin de facilitar la vida cotidiana [8]. Desde la perspectiva del conocimiento y la información, Gartner definió las ciudades inteligentes como "múltiples sectores que cooperan para lograr resultados sostenibles a través del análisis de la información contextual en tiempo real, compartida entre la información sectorial específica y los sistemas de tecnología operacional”.

Para [9], una SC integra la gente con sus recursos físicos. En ese sentido, la SC es un conjunto multidimensional compuesto por: i) Personas y hábitat: acciones relacionadas con el concepto más amplio de Smart Community, ii) Economía y gobernanza: acciones encaminadas a apoyar el desarrollo de planes de economía inteligente iii) Energía: acciones dirigidas a mejorar el rendimiento energético iv) Medio ambiente: acciones dirigidas a mejorar la calidad del aire interior y exterior, optimizando el consumo de elementos naturales, iv) y movilidad: acciones orientadas a disminuir el uso de vehículos privados y la implementación de soluciones sostenibles [10].

En términos generales el término SC incluye una visión utópica de una ciudad que resuelve la complejidad de sus realidades, produce riqueza, sostenibilidad y bienestar a través de la tecnología, creando nuevas relaciones entre la sociedad y la tecnología [11]. El ámbito de 
estudio de la SC ha estado activo en el campo académico, de tal forma que se podrían plantear hallazgos con respecto al área de conocimiento a partir del análisis de los documentos publicados en bases de datos indexadas y de alto impacto, la manera en que esto se desarrollará se hace explicito en el siguiente apartado.

\section{METODOLOGÍA}

Con el fin de conocer las tendencias y evolución de la investigación en SMART CITY (SC), se usa el enfoque de bibliometría apoyado por herramientas tecnológicas diseñadas para facilitar la visualización científica de información. Para desarrollar este análisis se hizo una búsqueda en la base de datos Web of Science (WoS) de Clarivate Analytics. De la búsqueda se extrajeron los metadatos que posteriormente fueron analizados por medio de la herramienta de análisis de redes bibliométricas VOSviewer.

La técnica de análisis que usa el software VOSviewer es la denominada VOS (visualization of similarities), para la construcción de mapas teniendo en cuenta información como la cocitación de autores y revistas y la coocurrencia de palabras clave y textos [12]. El término cocitación entendido como la asociación de artículos a causa de que ellos son citados de manera simultanea por otro artículo [13] y la coocurrencia como los términos que aparecen simultáneamente en un par de documentos.

De la búsqueda inicial, utilizando la sentencia de búsqueda "smart city", se obtuvo una muestra de 2833 documentos, en el periodo comprendido entre 2001-2019. En la Tabla 1 se observa de manera resumida la manera en que se aplicó la metodología de estudio en el presente artículo

Tabla 1: Resumen de implementación de la metodología del artículo.

\begin{tabular}{|c|c|}
\hline Fase & $\begin{array}{c}\text { Resumen } \\
\end{array}$ \\
\hline 1.Pregunta de investigación & ¿Cuál es la estructura conceptual del campo de investigación en SC? \\
\hline 2.Identificación de fuentes & Web of Science (WoS) \\
\hline 3. Extracción de metadatos & Periodo 2001-2019 (2833). Lotes de 500 registros completos con referencias citadas \\
\hline 4.Visualización de redes prueba & $\begin{array}{l}\text { Visualización de red de coocurrencia de palabras clave (2001-2019). } \\
\text { Mapa de red de ocurrencia de términos (2001-2019). }\end{array}$ \\
\hline $\begin{array}{l}\text { 5. Minería de datos y depuración } \\
\text { de registros }\end{array}$ & Construcción de tesauro para la depuración de 12 etiquetas duplicadas \\
\hline $\begin{array}{l}\text { 6. Definición de unidades de } \\
\text { análisis }\end{array}$ & Palabras clave indexadas por la base de datos (Keyword Plus) \\
\hline $\begin{array}{l}\text { 7. Definición de tipos de análisis } \\
\text { bibliométricos }\end{array}$ & Co-word análisis (Coocurrencia de palabras clave indexadas). \\
\hline $\begin{array}{l}\text { 8. Visualización de mapas de } \\
\text { redes (normalización, } \\
\text { distribución y clusterización) }\end{array}$ & $\begin{array}{l}\text { Parámetros estándar de visualización de VOSviewer para la normalización, distribución y clusterización. } \\
\text { Tamaño mínimo de los clústeres: } 20 \text { nodos }\end{array}$ \\
\hline
\end{tabular}

Fuente: Elaboración propia.

\section{IV.RESULTADOS AÁNLISIS E INTERPRETACIÓN}

Los metadatos obtenidos de la búsqueda de información en WoS fueron depurados de términos equivalentes para obtener los que se utilizarían como muestra de análisis. Las duplicidades encontradas corresponden a palabras plurales como "communities" la cual fue agrupada con "community" o "smart cities" con "smart city", esto fue posible a través de la implementación, a manera de tesauros, de la unificación de las etiquetas con el fin de que los nodos se unifiquen en los mapas de visualización de la red de conocimiento. Los metadatos depurados fueron base del análisis de coocurrencia de palabras clave, y coocurrencia de términos. Los mapas de red que ayudaron a visualizar la estructura del campo de conocimiento se generaron por medio del software de acceso libre VOSviewer [14]. La información en general es multidisciplinaria y a simple vista es difícil encontrar puntos en común para relacionarla entre si, por lo cual la visualización ayuda a crear una imagen mental de lo que no es obvio a simple vista. La visualización de información se ha vuelto una práctica común en las comunidades científicas, especialmente por la existencia de nuevas herramientas para este fin y como una manera de revelar la esencia del conocimiento científico [15].

La Figura 1, muestra el mapa de red de ocurrencia de términos basados en la información de texto. Es necesario mencionar que el análisis de esta información se hace por medio de minería de datos del texto contenido en el título y los resúmenes de los artículos dentro de la muestra [16]. Este proceso se hace en 2 etapas, inicialmente la identificación de los términos que se han de incluir en el mapa, identificando las fases sustantivas y de estas se hace una selección de los términos por medio de valores de relevancia, los cuales se determinan a través de la comparación de la distribución de coocurrencias entre las frases sustantivas [17], dándole más relevancia a términos que representan tópicos de investigación que a tópicos basados en términos generales. 


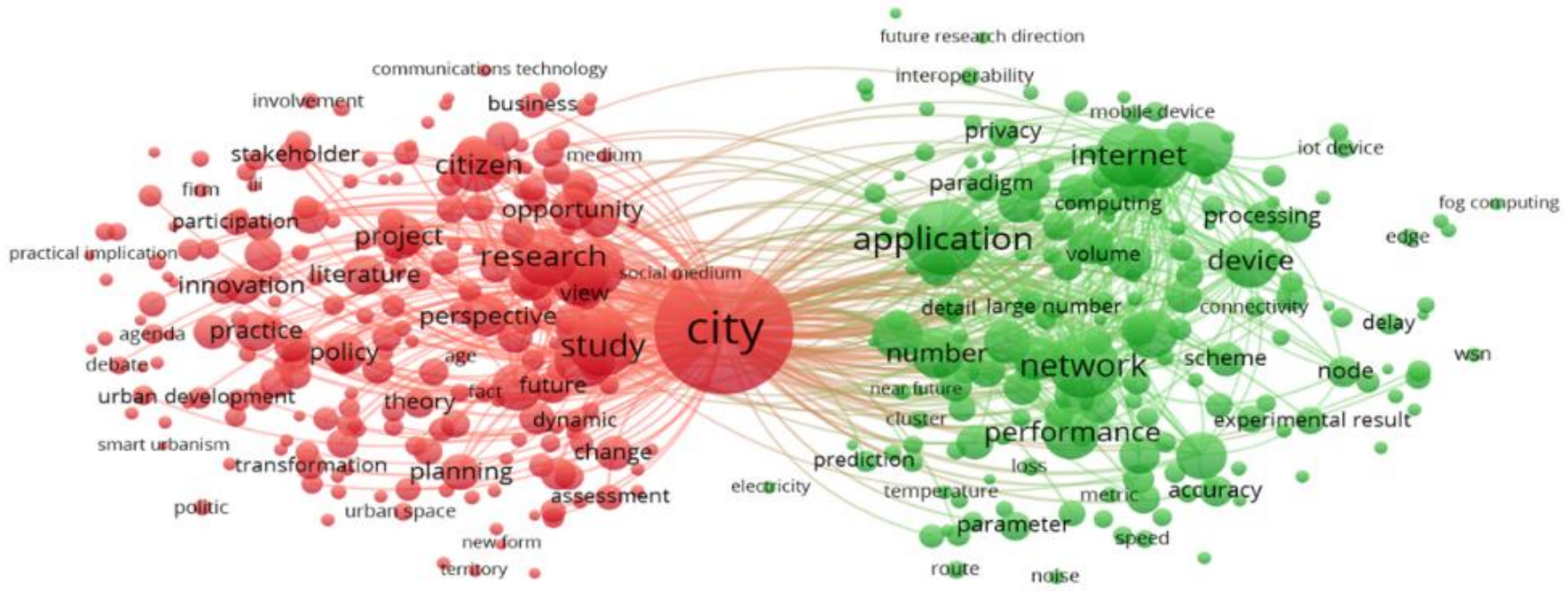

Figura 1: Mapa de red de ocurrencia de términos.

Fuente: Elaboración propia.

Para la visualización se tomaron los términos que tienen al menos 20 ocurrencias. El tamaño de los círculos representa el número de veces en que la palabra/frase aparece, siendo "city" la de mayor ocurrencia. Según los valores de relevancia, los términos Wireless sensor network (wsn)", "fog computing", "smart policy" y "Software-defined networking (sdn)", son los que encabezan la lista, poponiendose como nuevos focos de investigación y tendencias de interés de investigación. De la red de conocimiento se puede observar el termino "city" como eje central y se visualiza la separación de términos acorde a la finalidad de los estudios y las metodologías usadas para ese fin. En el caso del sector rojo se concibe la orientación de los estudios hacia la práctica, investigación, perspectivas, innovación, entre otros, mientras que en el sector verde aplicaciones de Smart city, rendimiento, internet, devices, entre otras, dan cuenta de los enfoques utilizados en el contexto de Smart city.

En la Figura 2, se observa el mapa de red de coocurrencia de palabras clave. La muestra de artículos analizados incluye 2579 palabras clave, de las cuales, como criterio de simplificación, se incluyen las palabras que tienen un mínimo de 10 ocurrencias, generando una red con 126 palabras clave, 2549 links y una fortaleza de links de 7404. En esta representación las etiquetas son representadas en círculos, el tamaño del circulo depende de la cantidad de ocurrencias (atributo que indica el número de documentos en que aparece la palabra clave), las cuales están conectadas por líneas que representan el vínculo entre etiquetas, el cual se mide a partir de las veces que son incluidas mutuamente en documentos de la muestra. La fortaleza de vínculo esta representada por el grosor de la línea, siendo más gruesa cuando la fortaleza es mayor y más delgada cuando la fortaleza es menor. Adicionalmente, para la construcción de los clústeres se estipuló un tamaño mínimo de clúster de 20 componentes, resultando tres clústeres en los cuales se puede observar que el área de conocimiento se focaliza en 3 temáticas: 1) Desarrollo de ciudades a partir de la innovación, adopción de tecnología y big data enfocados hacia la gobernanza 2) Retos de seguridad informática, privacidad y el diseño ciudades a través del internet e internet de las cosas y 3) la administración de las smart city por medio de modelos [17] y sistemas simulados.

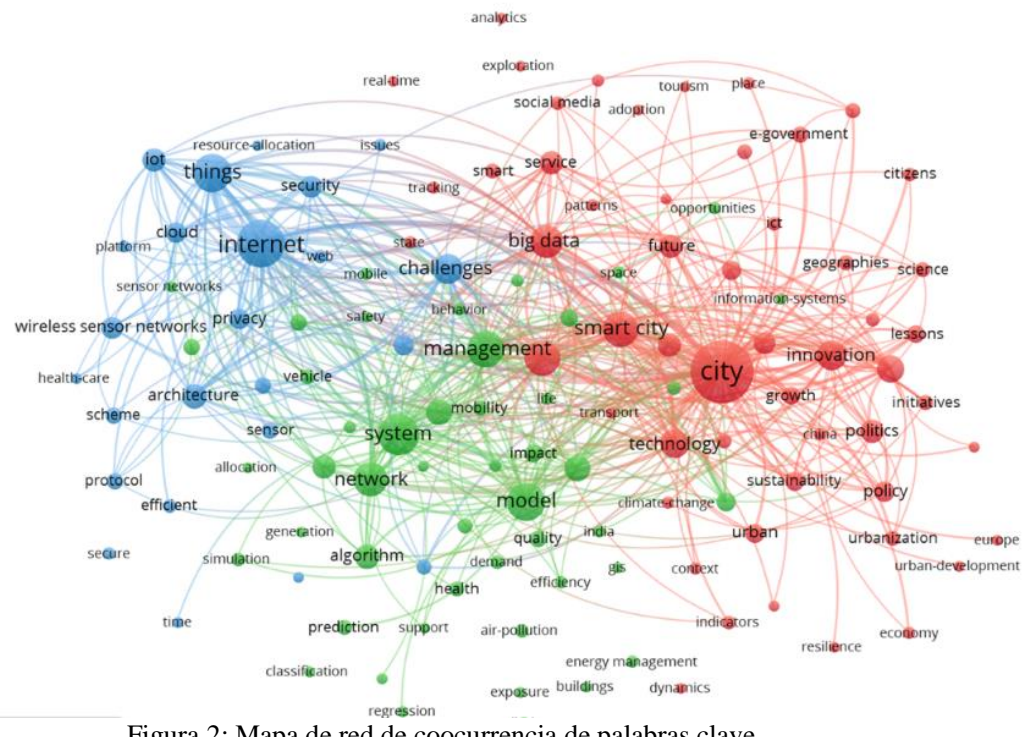

Figura 2: Mapa de red de coocurrencia de palabras clave.

Fuente: Elaboración propia.

En la Tabla 2, se detalla la tabla de coocurrencia de palabras clave. Se observan las 20 palabras que más veces han estado presentes en el keyword plus, siendo city con 583 la de mayor ocurrencia, seguido por Internet con 281, system con 192, model con 168 y things con 168. 


\begin{tabular}{|l|c|l|r|}
\hline Keyword & Occurrences & Keyword & Occurrences \\
\hline city & 513 & challenges & 101 \\
\hline internet & 281 & innovation & 93 \\
\hline system & 192 & technology & 90 \\
\hline model & 168 & governance & 83 \\
\hline things & 162 & performance & 69 \\
\hline management & 148 & design & 68 \\
\hline smart city & 143 & architecture & 60 \\
\hline framework & 138 & algorithm & 60 \\
\hline network & 128 & information & 58 \\
\hline big data & 118 & future & 55 \\
\hline
\end{tabular}

Fuente: Elaboración propia.

En la Tabla 3 se propone una matriz cruzada, en la cual se relacionan las 10 palabras con mayor fortaleza de vínculo medido a partir de las veces que aparece en conjunto con otras palabras. La palabra con mayor fortaleza de vínculo es city, con 1298 combinaciones, seguido por internet con 809, management con 532, things con 530 y system con 509. De la matriz cruzada se determina que city ha sido 57 veces utilizado en conjunto con la palabra internet, 50 veces con la palabra management, 47 veces con la palabra big data y 46 veces con las palabras model y framework. De esto se infiere que los estudios están concentrados en la administración de ciudades a través de internet y big data como marcos de desarrollo. Las palabras que más veces se usaron en conjunto como palabras clave son internet y things con 150 veces, debido a que estas palabras unidas forman toda un área de conocimiento.

Tabla 3: Matriz de análisis cruzado de palabras

\begin{tabular}{|l|c|c|c|c|c|c|c|c|c|c|}
\hline & city & internet & management & things & system & framework & model & big data & smart city & challenges \\
\hline city & 1298 & 57 & 50 & 36 & 33 & 46 & 46 & 47 & 33 & 32 \\
\hline internet & 57 & 809 & 32 & 150 & 26 & 29 & 16 & 21 & 17 & 29 \\
\hline management & 50 & 32 & 532 & 19 & 25 & 17 & 21 & 10 & 17 & 17 \\
\hline things & 36 & 150 & 19 & 530 & 12 & 17 & 11 & 18 & 12 & 20 \\
\hline system & 33 & 26 & 25 & 12 & 509 & 15 & 21 & 9 & 13 & 13 \\
\hline framework & 46 & 29 & 17 & 17 & 15 & 494 & 16 & 13 & 10 & 14 \\
\hline model & 46 & 16 & 21 & 11 & 21 & 16 & 466 & 12 & 11 & 8 \\
\hline big data & 47 & 21 & 10 & 18 & 9 & 13 & 12 & 425 & 16 & 14 \\
\hline smart city & 33 & 17 & 17 & 12 & 13 & 10 & 11 & 16 & 414 & 8 \\
\hline challenges & 32 & 29 & 17 & 20 & 13 & 14 & 8 & 14 & 8 & 393 \\
\hline
\end{tabular}

Fuente: Elaboración propia.

Se puede notar que existen otras relaciones que vale la pena mencionar, como es el caso de internet y management, que tienen una fortaleza de vínculo de 32 y que hace evidente la influencia mediada en internet en la gobernaza, y la relación de internet con challenges la cual delinea los retos que estan presentes en el desarrollo de la tecnología de internet como una de las dimensiones de la SC. La fortaleza de vínculo de framework supera a la fortaleza de vínculo de modelos, probando lo que menciona [11], que no existe una claridad en lo que respecta a los modelos que definan las SC, inclinándose los estudios hacia marcos de trabajo.

\section{CONCLUSIONES}

La descripción de áreas de conocimiento a través de la producción científica brinda una visión particular de las tendencias, oportunidades y temáticas desarrolladas por los investigadores. La bibliometría, a partir del análisis de minería de datos y de agrupación de palabras, facilita el entendimiento del conocimiento disponible, y la visualización de mapas facilita reconocer la ocurrencia, coocurrencia y fortalezas de vínculos entre palabras/frases utilizadas en un universo definido, que en este caso particular fue la base de datos WOS.

El término SC adquiere significado para enfrentar los problemas de unas sociedades complejas buscando a través de la tecnología crear bienestar, sostenibilidad y riqueza a sus habitantes. El análisis de relevancia propone como focos de investigación los términos "Wireless sensor network (wsn)", "fog computing", "smart policy" y "Software-defined networking (sdn)" las cuales se pueden denominar tendencias de interés de investigación.

Del análisis de clusterización resultan tres clústeres que enmarcan las tres principales temáticas extraídas de los metadatos así: 1) Desarrollo de ciudades a partir de la innovación, adopción de tecnología y big data enfocados hacia la gobernanza 2) Retos de seguridad informática, privacidad y el diseño ciudades a través del internet e internet de las cosas y 3) la administración de las smart city por medio de modelos y sistemas simulados.

Por medio de una matriz cruzada que relaciona las palabras con mayor fortaleza de vínculo se puede inferir que los estudios principalmente se han decantado en la administración de los recursos y las ciudades a través de internet y el uso de big data como marcos generales de trabajo.

\section{VI.RECOMENDACIONES}

El análisis bibliométrico incluye análisis adicionales como la coautoria, citación y cocitación, que se podrían utilizar para complementar los hallazgos, pero que en este momento están fuera del alcance de este. Se espera que para una próxima publicación se pueda incluir un análisis centrado en los lugares en los que se genera la investigación y en los autores, de tal forma que se ilustre el desarrollo del área de investigación a partir de los protagonistas de los escritos. 


\section{REFERENCIAS}

[1] J. Linares y K. Vásquez. «Ciudades inteligentes: ¿materialización de la sostenibilidad o estrategia económica del modelo neoliberal?», El Ágora USB, Revista de Ciencias Sociales, vol. 18, n² 2, pp. 479-495, 208.

[2] Deloitte, «Super Smart City - Happier Society with Higher Quality | Deloitte China | Public Sector», deloitte.com, 2018. https://www2.deloitte.com/cn/en/pages/public-sector/articles/super-smart-city.html\# (accedido mar. 27, 2020).

[3] R. A. López -Alvarado, «Ciudad Inteligente y Sostenible: hacia un modelo de innovación inclusiva», Paakat Rev. Tecnol. Soc., vol. 7, n.o 13, pp. 1-17, 2017.

[4] G. M. Martinez-Toro, D. Rico-Bautista, E. Romero-Riano, C. J. Galeano-Barrera, C. D. Guerrero, y J. A. Parra-Valencia, «Analysis-ofthe-intellectual-structure-and-evolution-of-research-in-human-computer-interaction-A-bibliometric-analysis», Rev. Iber. Sist. E Tecnol. Informaçao, vol. 17, pp. 363-378, ene. 2019.

[5] Y. He, I. Stojmenovic, Y. Liu, y Y. Gu, «Smart City», Int. J. Distrib. Sens. Netw., vol. 10, n.o 5, p. 867593, may 2014, doi: $10.1155 / 2014 / 867593$.

[6] A. Meijer and M. P. R. Bolívar, «Governing the smart city: a review of the literature on smart urban governance», Int. Rev. Adm. Sci., vol. 82, n.o 2, pp. 392-408, jun. 2016, doi: 10.1177/0020852314564308.

[7] A. Mattoni, F. Gugliermetti, and F. Bisegna, «A multilevel method to assess and design the renovation and integration of Smart Cities», Sustain. Cities Soc., vol. 15, pp. 105-119, 2015.

[8] R. Szabó et al., «Framework for smart city applications based on participatory sensing», en 2013 IEEE 4th International Conference on Cognitive Infocommunications (CogInfoCom), 2013, pp. 295-300.

[9] Y. Atif and S. Mathew, «A social web of things approach to a smart campus model», en 2013 IEEE International Conference on Green Computing and Communications and IEEE Internet of Things and IEEE Cyber, Physical and Social Computing, 2013 , pp. 349-354.

[10] G. M. Martinez-Toro, G. C. Ariza-Zabala, D. R. Bautista, and E. Romero-Riaño, «Human computer interaction in transport, a systematic literature review», en Journal of Physics: Conference Series, 2019, vol. 1409, n.o 1, p. 012002.

[11] A. J. Meijer, J. R. Gil-Garcia, and M. P. R. Bolívar, «Smart City Research: Contextual Conditions, Governance Models, and Public Value Assessment», Soc. Sci. Comput. Rev., vol. 34, n.o 6, pp. 647-656, dic. 2016, doi: 10.1177/0894439315618890.

[12] N. J. van Eck, L. Waltman, R. Dekker, and J. van den Berg, «A comparison of two techniques for bibliometric mapping: Multidimensional scaling and VOS», J. Am. Soc. Inf. Sci. Technol., vol. 61, n.o 12, pp. 2405-2416, dic. 2010, doi: 10.1002/asi.21421.

[13] E. Garfield, «From bibliographic coupling to co-citation analysis via algorithmic historio-bibliography: A citationist's tribute to Belver C. Griffith, Lazerow Lecture presented at Drexel University, Philadelphia, PA. November 27, 2001», PA Retrieved March, vol. 5, p. 2015, 2001.

[14] N. J. van Eck y L. Waltman, «VOSviewer Manual». Centre for Science and Technology Studies CWTS, ene. 27, 2020, [En línea]. Disponible en: https://www.vosviewer.com/documentation/Manual_VOSviewer_1.6.14.pdf.

[15] B. Vargas-Quesada y F. de Moya-Anegón, Visualizing the structure of science. Springer Science \& Business Media, 2007.

[16] N. J. van Eck y L. Waltman, «Text mining and visualization using VOSviewer», p. 5.

[17] G. M. Martinez-Toro, G. C. A. Zabala, y A. R. Piedrahita, «El rol de los modelos en el aprendizaje organizacional y el diseño de políticas», Rev. Ibérica Sist. E Tecnol. Informação, n.o E28, pp. 386-398, 2020. 